of knife, wrapper, butter, or milk could be made with other regiments. The regiments of the 1st Brigade were all close to one another in camp when most of the cases occurred and from August 24th only some four or five had not heen present in that camp. Though the cheese doubtless killed the sheep, more evidence is required to definitely settle the cause of the disease amongst the men. Having dealt with all other articles of food about which we could obtain information and as we are both under orders to proceed shortly on active service to South Africa, we publish these particulars with a hope that some of the readers of THE LANCET may be able to throw further light on the subject.

Aldershot.

\section{A CASE OF INFECTIVE ENDOCARDITIS TREATED BY ANTI-STREPTOCOCCIC SERUM, NUCLEIN, \&C. ; DEATH.}

BY R. HINGSTON FOX, M.D. BRUX., M.R.C.P. LOND., AND

E. AUGUSTUS LERMitTE, M B., B.S. DuRh., L.R.C.P. LOND., M.R.C.S. ENG.

A MAN, aged 39 years, consulted Dr. Hingston Fox on Jan. 4th, 1899 , for pain in the lambar spine, with tingling in the legs and feet after exertion. The patient was a delicate-looking man, of anxious temperament, and had suffered from influenza with rheumatic symptoms a year previously. No history of exposure to sewer gas or other septic conditions could be made out. His father died at 51 years of age from carbuncle and his mother died at the age of 76 years, but she had had delicate lungs in early life. There was much tenderness over the whole lumbar spine, the kneejerks were active, and there was some ankle clonus on the left side. The pain was iessened by rest, but it did not pass away, and as the tenderness on pressure continued and there was a slight deviation of the lower part of the lumbar spine to the left, it was thought on Feb. 9th that caries of the spine was impending. The urine contajned albumin, at first a trace only, but increasing week by week, with excess of acidity, much deposit of urates, many squamous, mucous, and renal cells, and a few small casts. On one occasion a good many tufts of blood-vessels were found in the urinary sediment, with capsules apparently containing cells. A small rough systolic bruit was heard over the aortic orifice on Feb. $9 \mathrm{ch}$. The patient was confined to his bed and couch from about Feb. 10th and the actual cautery was applied near the spine on four occasions with the result that the spinal pain entirely subsided. Remittent pyrexia (from about $99^{\circ} \mathrm{F}$. to $101^{\circ}$ or $102^{\circ}$ ) was observed from his first confinement to bed and continued thenceforth. The urine continued to be albuminous and loaded with urates and uric acid, the latter sometimes in a copious sediment. Pain and swelling, apparently brief inflammatory embolisms only lasting a few hours or a day or two, appeared during the latter half of March in various locations-the right wrist, the left cheek, and the left testicle. The temperature rose higher in the last week of March and from that time pursued an irregular, fluctuating course, seldom falling below $100^{\circ}$ in the morning and generally reaching $102^{\circ}$ or $103^{\circ}$ in the evening. There were frequent sweats but no rigors. Meanwhile tbe heart became gradually dilated and double aortic and systolic mitral bruits developed. Staphylococci, probably pyogenes albus, were found in the blood on April 5 th, but no streptococci. The staphylococcus pyogenes aureus was obtained from pustules on the $25 \mathrm{th}$. Dr. Lermitte took charge of the case on April 6th, Dr. Fox continuing to see the patient in consultation at intervals. Signs appeared of much effusion into, the peritoneal and pericardial cavities and some into the pleural cavities. There were varying evidences of pneumonia and pleurisy at the bases of the lungs and during the last month of life of hypostatic congestion, also some aphthous stomatitis. The liver and spleen were enlarged and there were pains in the jaws, shoulders, and other joints. The patient died on May 10th from slow heart failure with attacks, shortly before the end, of pain in the heart region and in the back of the thorax. The morning fall of temperature extended much below normal in the latter five days of life. The patient took food well throughout, but became wasted and anæmic.

At the necropsy the heart was found to be much enlarged and the left ventricle was distended and filled with firm dark clot. The aortic valves were distorted and covered at their edges and bases by abundant granulations and there were some granulations also on the auricular surfaces of the mitral valves. The pericardium contained about four ounces of turbid bloody fluid containing tlakes. The right pleura was more or less adherent throughout, especially to the diaphragm, and the left pleural cavity contained some fluid. Both lungs were gorged with blood, with signs of recent pneumonia at the bases. There was some cicatrisation at both apices, including at the right apex calcareous deposit. The peritoneal cavity contained fluid. The liver and spleen were enlarged. The kidneys were also large, the capsule splitting into two layers, of which the inner was adherent; the cortex was pale and cloudy, the pyramids were dark, and many little red crystals of uric acid lay in the calices. There were no cysts or hæmorrhages. The lumbar inter-vertebral discs appeared to be healthy. No sign of suppuration was found anywhere in the body.

The treatment pursued was in part general and symptomatic, but the following points are of interest. It was strongly suspected that a suppurative lesion was present, and on April 9th the patient was thoroughly examined under an avæsthetic and the needle was inserted in several parts of the loins and back, but no pus was fourd. Quinine was given for about two weeks in three-grain or fuur-grain doses, or two grains three times a day, but it was wholly without effect on the temperature. Sodium salicylate and alkalies seemed to reduce it sometimes. Arsenic was also used for some weeks Anti-streptococcic serum from the Jenner Institute was obtained through Messrs. Allen and Hanburys and used assiduously from April 4th to 11th. Fifteen doses in all were injected beneath the skin of the abdomen or arm, generally of $10 \mathrm{cubic}$ centimetres each, and amounting to a total of 149 cubic centimetres. The local irritation was considerable and the patient disliked the injections; eventually copious erythematous lichen developed over much of the abdomen and the arm, as well as bosses of erythema at the site of the injections. The temperature, at that time ranging between $100^{\circ}$ and $103^{\circ}$, was in no way lowered, perhaps it was a little raised, uuder their use, and be was unusually drowsy There was no remission of any symptom. Nuclein was then tried from the 12 th to the 16 th, 11 hypodermic injections being used, at tirst of from 20 minims to 30 minims of an old 1 per cent. solution made by Messrs. Burroughs and Wellcome and later 16 minims of a new solution of the same strength prepared on purpose for this case by the same firm. The injections caused pain for a minute or two, the liquid evidently irritating the tissues, and a very itchy rash came out over the abdomen and the back. The temperature remained high and no good effect whatever could be observed. The inhalation of oxygen was used during the latter weeks from time to time and the pulse-rate was quieted for awhile thereby-e g., from 130 to 112 Small doses of antifebrin were also given during two days. On April 19th and 29th Dr. Lermitte applied an eclectic German oil, apparently containing croton oll or some similar drug. The chest and abdomen were first covered with rows of fine multiple punctures by a scarificator and the oil was painted over them, a thick wool wrapping being immediately applied and kept closely in apposition. In the course of two or three days a crop of innumerable small pustules had developed, and these in another day or two dried up and the scabs could be brushed away. In the meantime the patient was very thoroughly fed. The treatment was borne better than might be supposed and gave very little trouble. Under the first application tice temperature steadily declined for four days, the minima and maxima being as follows: April 19th, $1004^{\circ}$. $103^{\circ}$; Z0th, $998^{\circ}, 101 \cdot 8^{\circ} ; 21$ st, $99^{\circ}, 101^{\circ} ; 22 \mathrm{ad}, 99^{\circ}, 1004^{\circ}$ and $23 \mathrm{rd}, 972^{\circ}, 100^{\circ}$. The pulse dropped from 120 to about 90 , the tongue cleaned, the urine increased in quantity, and the general condition of the patient was easier. After the fifth day the conditions all relapsed, the temperature reaching $1036^{\circ}$ on the 25 th and the pulse being 120 , and no further good effect could be traced. The second application was made at a late period when the heart was obviously failing and no distinct benefit was observed to follow it.

Remarks by Dr. HINGSTON Fox.-The origin of the specific infection in this case-cne of infective endocarditis of the T 2 
malarial or cardiac form-was not clearly shown; but the influence of a previously damaged aortic valve in the production of the disease was manifest, a fact to be remembered in life assurance examinations. The case unhappily progressed from the first to its fatal end little influenced by the treatment used. The record as to the efficacy of anti-streptococcic serum and nuclein is therefore a negative one, but inasmuch as these remedies received a thorough trial the facts are now published. So far as the experience of a single case goes it points to the uselessness of the serum when no streptococci, but only staphylococci, are found in the blood. In connexion with this it may be noted that a Russian physician has reported a case of malignant endocarditis treated successfully with antistaphylococcic serum. ${ }^{1}$ The marked, although temporary, benefit derived from free minute pustulation is noteworthy and brings to mind the "counter-stimulant" use of tartar emetic which was introduced by Dr. Edward Jenner and so extensively practised for all inflammatory diseases during the earlier half of this century. In the present case there were micrococci, toxins, and much hot inflammatory material circulating in the blood, and those who watched the patient during the pustulation and noted his declining pyrexia and general increased comfort for the time being, could not resist the inference that the pustules actually withdrew some of the noxious matter from the circulation. The patienc had also the advantage of the advice of Sir William Gowers, of Dr. Arthur Davies, and of Mr. A. H. Tubby, who saw him at various stages of his illness. The bacteriological examinations were made by the Jenner Institute of Preventive Medicine and the Clinical Research Association, whose reports agreed very closely.

\section{A CASE OF PREGNANCY OCCURRING IN A PATIENT WITH A DOUBLE UTERUS AND A DOUBLE VAGINA.}

\section{By C. J. GLASSON, M.D. BRUX, L.R.C.P. LoND.,} M.R C.S. ENG.

IN reporting this case I have done so that it may add to the few cases which are on record.

On the evening of Sept. 3rd, 1899, I was called to attend a primipara, aged 21 years, in her confinement. On my arrival at the house I found that she had been in labour for some hours and that the pains were getting very strong, but that the child did not seem to get any lower. The liquor amnii had burst some time before during one of the pains. The woman was walking about when I got to the house. I placed her on the bed and proceeded to examine her in order to find out the presentation, but to my surprise I could not feel the child's head exc oting through a thick membrane. At first I thought that I must have made a mistake and inserted my finger in the rectum, but I could feel the anus distinctly with my left index finger. I got my finger as high as I could possibly get it, but could not feel the os or the uterus. I withdrew my finger and re-inserted it; this time there was no mistake, for I could feel the child's head distinctly in the first position. The head had come through the os and was pressing down hard upon a thick band. There was no deformity of the pelvis either at the inlet or the outlet; in fact, there was plenty of room. I proceeded to examine her more carefully by the aid of a good lamp and upon separating the folds of the vulva I could distinctly feel and "see" a thick septum extending anteriorly and posteriorly across the vagina. I easily passed $m y$ index finger into the right side but felt no os, and again into the left vagina and felt the child's head. I passed my finger up along the whole of the septum until I came to its junction with the uterus. All the parts were, of course, congested and swollen as is usual at labour. While I was examining her during one of the pains, which were now becoming exceedingly powerful, I could feel the child's head pressing upon and throwing the septum into a fold which by stretching across the head prevented its coming down any further. Just before this pain tinished the patient complained that something had given way inside her and the pain suddenly ceased. I found, upon examination, that the upper portion of the septum had given way and that there was now a foramen through which

1 Brit. Med. Jour. Epitome, July 2nd, 1898, art. 12.
I could pass my finger into the right vagina. When the next pain came on I had been holding the septum to the right side of the vagina, so that, if possible, the child's head might slip through. This I was enabled to manage after a good deal of manipulation and had the satisfaction of getting the head safely past the obstruction. Soon the child's head was weli down on the perineum and while a stronger pain than usual was on the patient felt something more give way. This was the septum at its lower attachments. After that $I$ had no further trouble. The head was born, then the shoulders and the rest of the body. The child was a big male child and weighed, I should judge, between seven and eight pounds. There was a distinct mark across the head where the fold of the septum had pressed. I expressed the placenta and there was very little hæmorrhage. I examined the patient carefully after the placenta had come away and I could feel and see where the septum had been torn away and could feel the septum still whole up to the uterus except where the hole had been torn through close to the os. I could also feel high up on the right side a mass but could not make out definitely what it was. The perineum itself was intact. The patient made a splendid recovery and got up on the twelfth day.

I questioned the patient's mother concerning her, but she said that she had never noticed anything wrong. I asked the patient about her menses; these, she said, had been irregular, they had occurred once in tive or six weeks, and then they had been very scanty and had lasted about two days. She never menstruated after marriage and the baby was born 10 months after that event. I asked if ber husband had had any difficulty during intercourse. She replied in the negative, but that sometimes there had been some apparent obstruction, but of this she bad taken no notice. Four weeks after labour I made a thorough examination. Externally the vulva and labia were normal. The urethra, the anus, and the rectum were normal, but I could easily see by separating the labia where the septum had been attached in front along the median line at the base of the bladder and behind to the median line in front of the rectum, and on tracing the remains up I could feel where it became continuous with the part of the septum that was still whole. On passing the finger up into the left vagina I could feel the uterus which had regained its normal size, and bimanually I could feel the fundus which was pointing to the left side. I then passed my finger into the right vagina and there I found another uterus of about the size of the first one with its fundus pointing to the right side and between the two I could pass my finger up and touch my fingers placed externally. I could only feel one ovary on each side and none between. I did not examine her per rectum. The following diagram will explain the conditions found. I should add that the vaginal septum passed upward and was continuous with the septum between the two uteri.

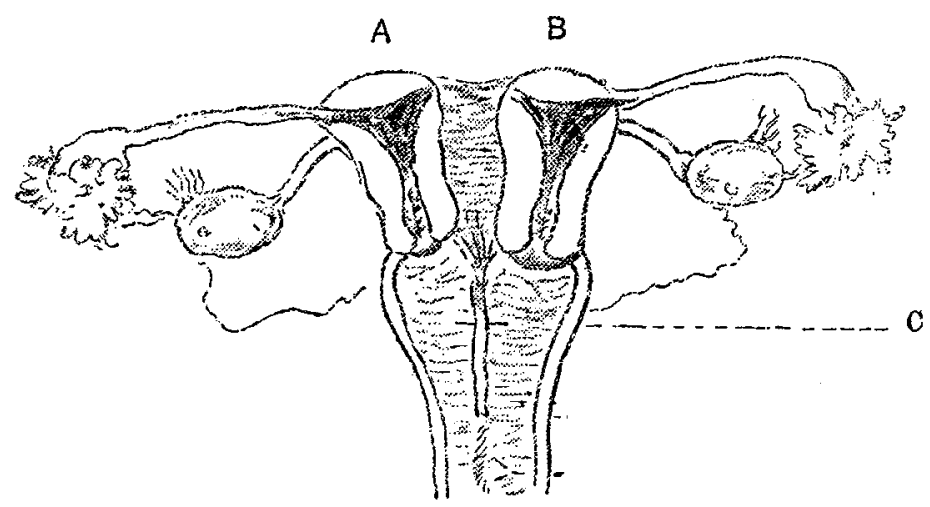

A, right uterus ; $B$, left uterus, in which pregnanuy occurred. C, Foramen at upper part of septum.

This is a rare case of abnormal development and one in which very serious trouble might bave arineu in the course of labour. Had there not been plenty of room to manipulate the band formed by the septum it would have had to be divided. I was exceedingly glad that I had to do no such thing, partly on account of the hæmorrhage that might have followed and partly on account of the smallness of the space to work in and the damage which might have been done by the knife when the pains were so frequent and of such a strong character. I have been trying to persuade the patient to undergo an operation for the removal of the septum, but neither she nor her husband will consent to anything being done, so for the present the conditions must remain in statu quo.

Chadwell Heath, Essex. 